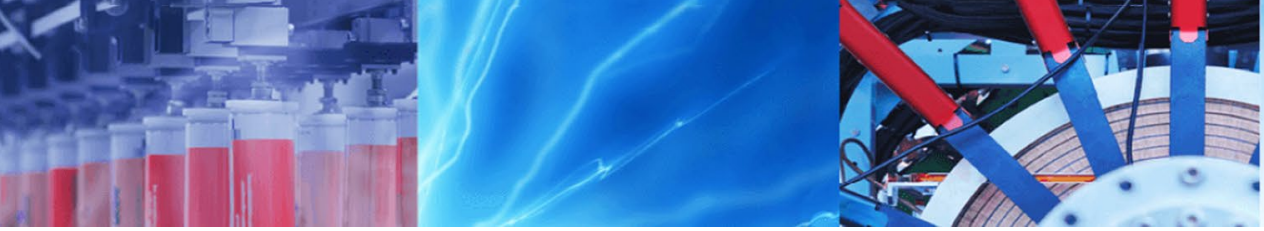

Short Communication

\title{
Fish bone-derived interconnected carbon nanofibers for efficient and lightweight microwave absorption
}

\author{
Yangyang Gu${ }^{1} \cdot$ Peng Dai ${ }^{1}$ [ $\cdot$ Wen Zhang ${ }^{1} \cdot$ Zhanwen $\mathrm{Su}^{1}$
}

Received: 25 November 2020 / Accepted: 12 January 2021 / Published online: 22 January 2021

(C) The Author(s) 2021 OPEN

\begin{abstract}
In this work, we demonstrated a simple method for preparing three-dimensional interconnected carbon nanofibers (ICNF) derived from fish bone as an efficient and lightweight microwave absorber. The as-obtained ICNF exhibits excellent microwave absorption performance with a maximum reflection loss of $-59.2 \mathrm{~dB}$ at the filler content of $15 \mathrm{wt} \%$. In addition, the effective absorption bandwidth can reach $4.96 \mathrm{GHz}$ at the thickness of $2 \mathrm{~mm}$. The outstanding microwave absorption properties can be mainly ascribed to its well-defined interconnected nanofibers architecture and the doping of nitrogen atoms, which are also better than most of the reported carbon-based absorbents. This work paves an attractive way for the design and fabrication of highly efficient and lightweight electromagnetic wave absorbers.
\end{abstract}

Keywords Biomass · Interconnected carbon nanofibers · Microwave absorption · Highly efficient

\section{Introduction}

With the rapid development of electronic technology, the problem of electromagnetic radiation pollution has become prominent and cannot be ignored [1, 2]. Thus, it is urgent to develop highly efficient electromagnetic wave absorption materials. Among various absorbents, $\mathrm{Fe}_{3} \mathrm{O}_{4}$ is extensively studied due to its low cost, high Curie temperature, unique dielectric/magnetic dual-loss properties, and environmental benignity [3, 4]. However, the high weight density and relatively low impedance matching restricts its application prospect. Recently, carbonaceous materials with unique structures have attracted tremendous attention for their low density, high electrical conductivity, large specific surface area, and controllable dielectric loss [5-8]. Quan et al. designed the macrostructure of the carbon nanotube dielectric filler and achieved ultra-high microwave absorption bandwidth [10]. Zhang et al. prepared the $\mathrm{MoS}_{2}-\mathrm{RGO}$ composite by a one-step hydrothermal method, which exhibited strong microwave absorption properties [10]. Despite those advantages, the fabrication route is complicated and expensive, which constrains their scalable applications. Therefore, it is still a challenge to synthesize carbon materials with outstanding microwave absorption properties through a low-price and simple approach.

Herein, fish bone, a typical kind of biomass waste, is used to prepare three-dimensional (3D) interconnected carbon nanofibers (ICNF) using a facile and simple process. It is well known that fish bone is cheaply obtained and mainly comprised of hydroxyapatite and collagen [11, 12]. Thus, fish bone can be used as the hard templates to synthesize ICNF with 3D interconnected framework architectures by a simple pyrolysis treatment and the following acidizing process. To the best of our knowledge, there has been no report on the fabrication of 3D interconnected carbon nanofibers from fish bone. The as-obtained ICNF exhibits excellent microwave absorption performance, and the minimum reflection loss is as high as $-59.2 \mathrm{~dB}$ at $7.92 \mathrm{~Hz}$ with a low filler percent of $15 \mathrm{wt} \%$. Moreover, the

Peng Dai, daipeng@mail.ustc.edu.cn | 'School of Physics and Materials Science, Anhui University, Hefei 230601, People's Republic of China. 
effective bandwidth can reach $4.96 \mathrm{GHz}$ when thickness is $2 \mathrm{~mm}$. These encouraging performances indicate that the as-prepared sample ICNF has great potential as an efficient and lightweight electromagnetic wave absorber.

\section{Experimental details}

\subsection{Synthesis of ICNF}

The typical process of synthesizing ICNF from fish bone is as follows. $10 \mathrm{~g}$ of fish bone powders was added into $80 \mathrm{~mL}$ of deionized water and stirred for $30 \mathrm{~min}$ at room temperature. Then, the above mixture was poured into a Teflon-lined autoclave for a hydrothermal treatment at $180^{\circ} \mathrm{C}$ for $10 \mathrm{~h}$. Subsequently, the precipitates were dried, grinded, and put into a horizontal tube furnace at $\mathrm{N}_{2}$ atmosphere with a heat treatment at $850^{\circ} \mathrm{C}$ for $2 \mathrm{~h}$. After that, the obtained black powders were soaked in $2 \mathrm{M}$ of $\mathrm{HCl}$ solution for $24 \mathrm{~h}$ to remove inorganic substances. Finally, ICNF powders were obtained after the treatment of centrifugation, washing and drying for use.

\subsection{Characterization of the samples}

The morphologies were observed by scanning electron microscopy (SEM, S-4800) and transmission electron microscopy (TEM, JEM-2100). The crystallinity was characterized by X-ray diffraction (XRD, Bruker D8-ADVANCE) with $\mathrm{Cu}$ Ka radiation. The specific surface area of samples was examined by the nitrogen adsorption-desorption isotherms (3H-2000PS2, BeiShiDe) using the Barrett-Emmett-Tellter (BET) method, and the pore diameter was calculated by the data of the adsorption branch of the $\mathrm{N}_{2}$ isotherm plot. The Raman spectrometer (inVia, Renishaw) was used to collect the Raman spectroscopy. The microwave absorption properties of the synthesized sample ICNF were measured by a vector network analyzer (Agilent N5071C, USA) in the frequency range of $2-18 \mathrm{GHz}$. Sample ICNF was homogeneously distributed in paraffin wax and pressed into a toroidal ring with the thickness of $2 \mathrm{~mm}$, outer diameter of $7 \mathrm{~mm}$, and inner diameter of $3.04 \mathrm{~mm}$, respectively.

\section{Results and discussion}

The morphology and microstructure of sample ICNF were characterized by SEM and TEM. As presented in Fig. 1a, sample ICNF exhibits 3D interconnected nanofibers architecture with the diameter in the range of $10-50 \mathrm{~nm}$ and the length of a few micrometers. Figure $1 \mathrm{~b}$ gives the TEM image of sample ICNF, which also confirms the unique 3D interconnected nanofibers network structure. The internal structure of sample ICNF is analyzed by XRD patterns, as shown in Fig. 2a. There are two broad and weak diffraction peaks at about $24.5^{\circ}$ and $43.4^{\circ}$, which can be well ascribed to the (002) and (100) planes of graphitic carbon, respectively, demonstrating the low graphitization degree of sample ICNF [13]. Raman spectroscopy is measured to further investigate the graphitization degree of sample ICNF. Figure $2 \mathrm{~b}$ shows two typical peaks centered around 1351 and $1590 \mathrm{~cm}^{-1}$, consistent with the features of amorphous carbon [14].

The specific surface area and porous structure of sample ICNF are evaluated by nitrogen adsorption-desorption measurements. As depicted in Fig. 3a, sample ICNF possesses a typical IV-type isotherm curve with a H3-type hysteresis loop, suggesting the existence of mesopores [15]. Based on the adsorption branch of $\mathrm{N}_{2}$ isotherm curve, the pore size distribution curve is calculated and illustrated in Fig. 3b. It is clear that the pore size of sample ICNF is mainly located in the range of 5-45 nm, also certifying the presence of mesopores. Moreover, sample ICNF has a large specific surface area value of $881 \mathrm{~m}^{2} \mathrm{~g}^{-1}$. The high specific surface area and the unique 3D interconnected nanofibers network architecture are suggested to be beneficial to the impedance matching, multiple microwave reflection, and interfacial polarization. Therefore, the as-synthesized sample ICNF has great potential in terms of microwave
Fig. 1 a A typical SEM image, and $\mathbf{b}$ a typical TEM image of sample ICNF
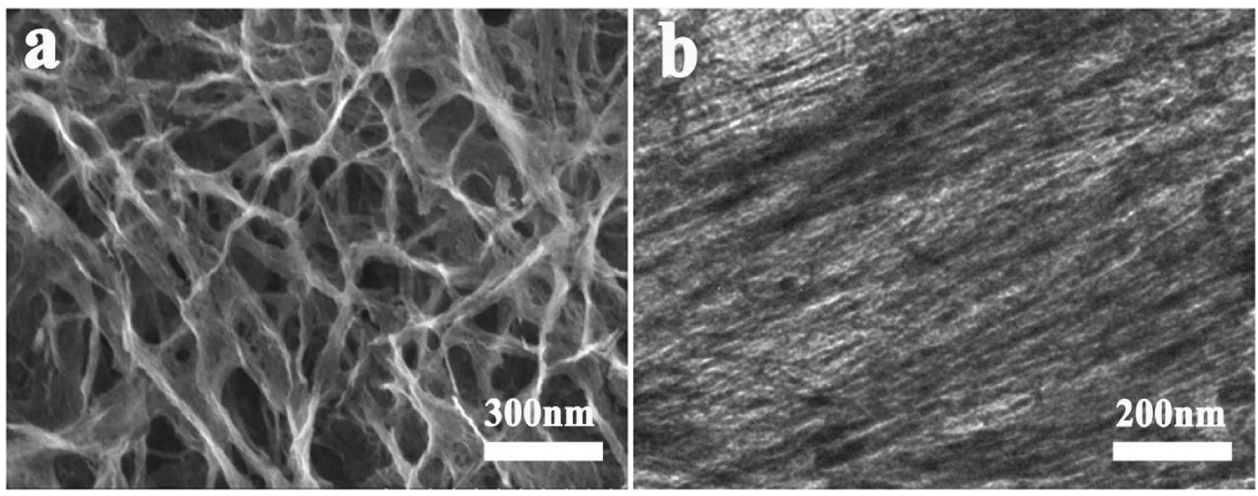
Fig. 2 The XRD pattern a, and the Raman spectrum $\mathbf{b}$ of sample ICNF
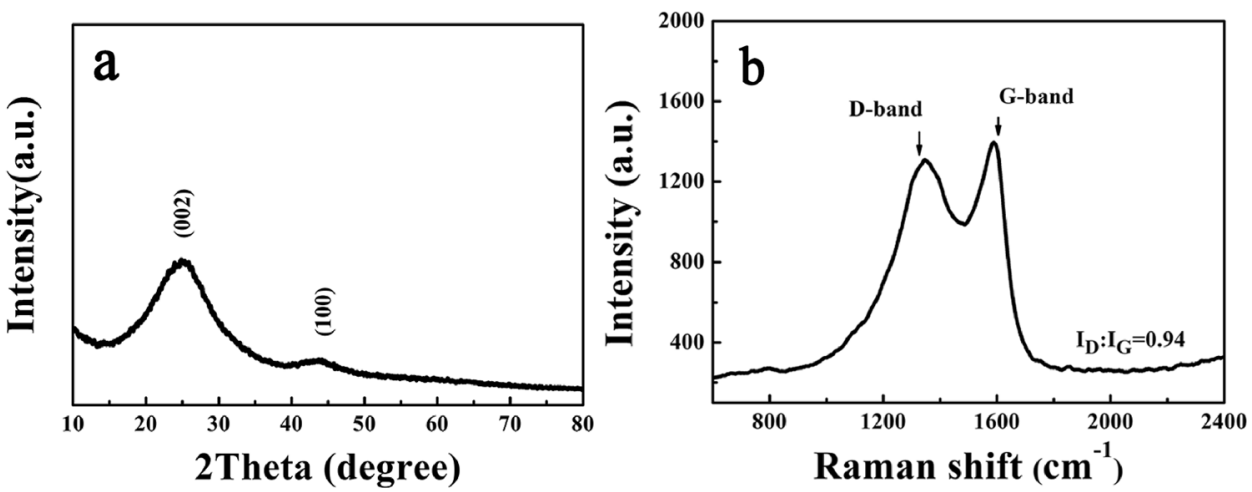

Fig. $3 \mathrm{~N}_{2}$ absorption-desorption isotherm (a), and the pore size distribution (b) of sample ICNF. XPS spectra of sample ICNF: C 1 s (c), and N 1 s (d)
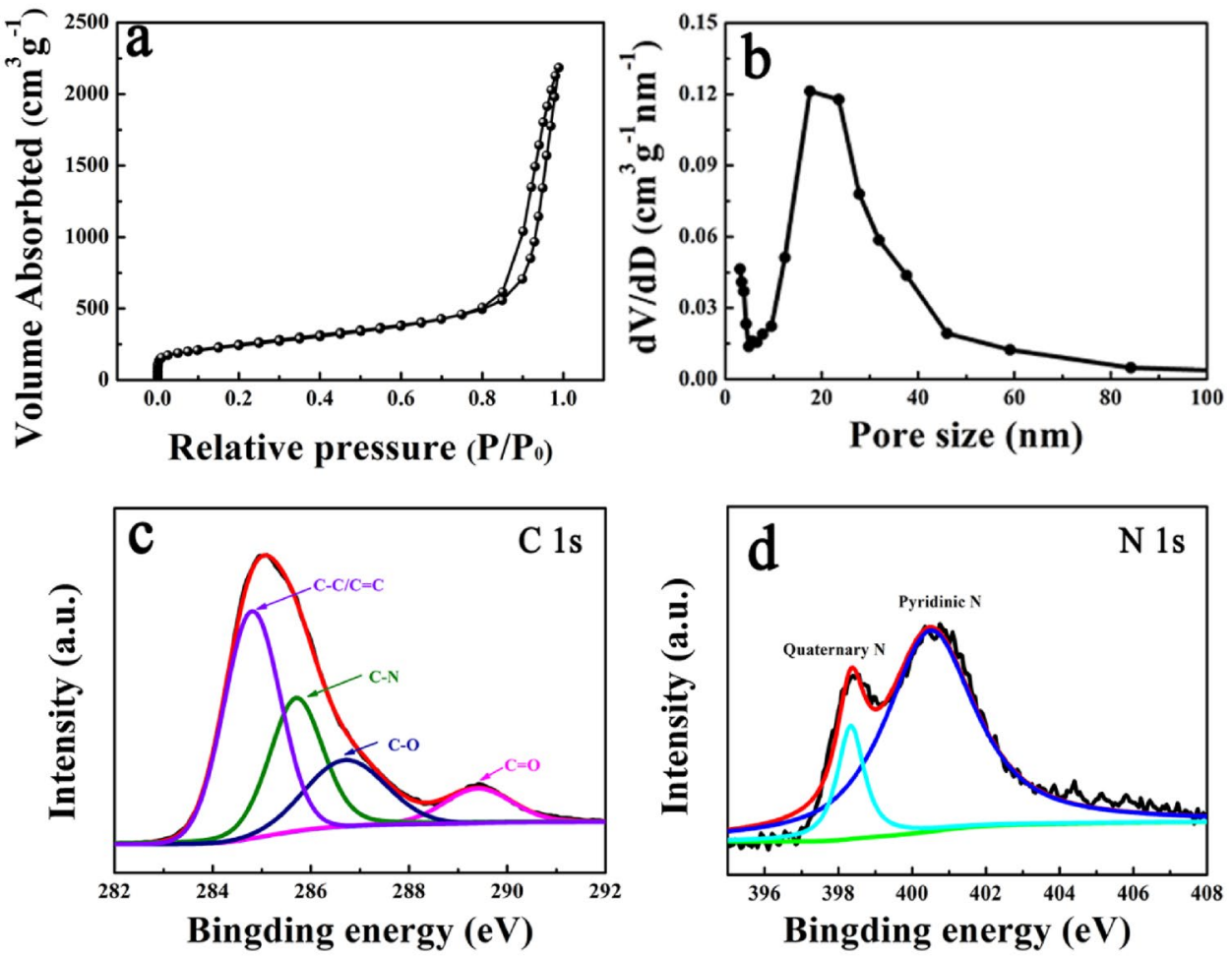

absorption. In addition, the surface composition and chemical state of sample ICNF were determined by the XPS technique. The $C 1 \mathrm{~s}$ spectrum in Fig. $3 \mathrm{c}$ can be deconvoluted into four peaks around 284.8, 285.7, 286.7, and $289.4 \mathrm{eV}$, which are attributed to $\mathrm{C}-\mathrm{C} / \mathrm{C}=\mathrm{C}, \mathrm{C}-\mathrm{N}, \mathrm{C}-\mathrm{O}$, and $C=O$, respectively $[16,17]$. For $N 1 \mathrm{~s}$ spectrum in Fig. $3 d$, it demonstrates that the binding energies around 398.3 and $400.5 \mathrm{eV}$ are assigned to pyridine $\mathrm{N}$ and quaternary $\mathrm{N}$, respectively $[18,19]$. The XPS analysis confirms the presence of nitrogen atoms in sample ICNF, which is suggested to be conductive to the dielectric loss and microwave absorption performance.

The microwave absorption performance is evaluated by the complex permittivity $\varepsilon$ due to the fact that no magnetism exists in sample ICNF. The real part $\varepsilon^{\prime}$ and the imaginary part $\varepsilon^{\prime \prime}$ of as-fabricated ICNF/paraffin composites with different absorbent loading are depicted in Fig. 4a and Fig. 4b, which represent the storage and dissipation capability of electromagnetic wave energy, respectively. For the filler ratio of $15 \mathrm{wt} \%$ shown in Fig. 4a, the $\varepsilon^{\prime}$ value decreases with increasing frequency. While its $\varepsilon^{\prime \prime}$ value in Fig. $4 \mathrm{~b}$ firstly reduces slightly in the range of $2-11 \mathrm{GHz}$, then increases dramatically in the frequency range of 11-18 GHz with obvious fluctuation and a maximum peak at $17 \mathrm{GHz}$, which might be from the polarization relaxation process occurring in sample ICNF. In addition, with the increase in loading content from 10 to $20 \mathrm{wt} \%$, the values of $\varepsilon^{\prime}$ and $\varepsilon^{\prime \prime}$ firstly increase and then decrease. Thus, there is an optimal content of ICNF, only the proper ICNF loading in ICNF/paraffin can get the best complex permittivity performance. The dielectric loss tangent $\left(\tan \delta_{\varepsilon}=\varepsilon^{\prime \prime} / \varepsilon^{\prime}\right)$ is further calculated to assess dielectric loss capability of ICNF/ 
Fig. 4 The relative permittivity real part $\mathbf{a}$, the relative permittivity imaginary part $\mathbf{b}$, the dielectric loss tangent $\mathbf{c}$, and the Cole-Cole semicircles $\mathbf{d}$ of ICNF/paraffin composites with different absorbent loading
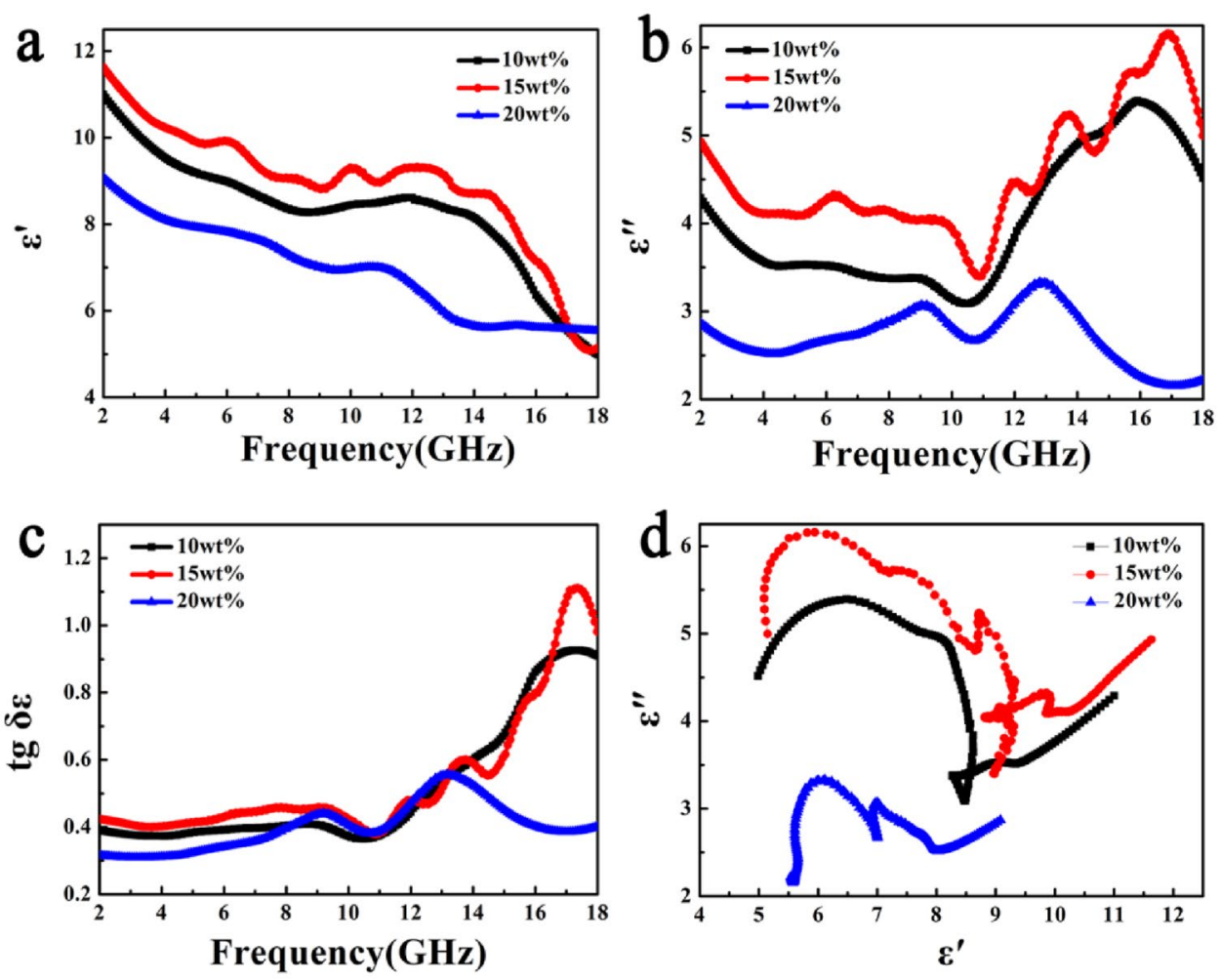

paraffin composites. As illustrated in Fig. 4c, the ICNF/paraffin with $15 \mathrm{wt} \%$ filler content also has the highest value of $\tan \delta_{\varepsilon^{\prime}}$ which maintains almost unchanged around 0.4 in the range of 2-11 GHz, then increases sharply along with increasing frequency and has a maximum peak at $17 \mathrm{GHz}$, suggesting the occurrence of intense dielectric loss effect. Moreover, the Cole-Cole plots of ICNF/paraffin composites are applied based on the Debye relaxation theory to better investigate the polarization relaxation behaviors. As shown in Fig. 4d, there are more distorted Cole-Cole semicircles with a tail in $15 \mathrm{wt} \%$ ICNF/paraffin composite, suggesting the existence of more forms of relaxation process.

According to transmission line theory, the reflection loss $(\mathrm{RL})$ values of as-synthesized samples are calculated based on the following formula to evaluate the electromagnetic wave absorption properties $[20,21]$.

$\mathrm{RL}(\mathrm{d} B)=20 \log \left|\frac{Z_{\text {in }}-Z_{0}}{Z_{\text {in }}+Z_{0}}\right|$

$Z_{\text {in }}=\sqrt{\frac{\mu_{r}}{\varepsilon_{r}}} \tanh \left[j\left(\frac{2 \pi f d}{c}\right) \sqrt{\mu_{r} \varepsilon_{r}}\right]$

where the parameters $Z_{\mathrm{in}}, Z_{0}, f, d$, and $c$ are input impedance, free space impedance, applied frequency, absorber thickness, and light velocity, respectively. Figure $5 \mathrm{a}-\mathrm{c}$ exhibits the three-dimensional patterns of ICNF/paraffin composites with different ICNF loading at different thickness. When filler ratio of ICNF is $15 \%$ (Fig. $5 b$ ), the minimum value of $\mathrm{RL}$ is up to $-59.2 \mathrm{~dB}$ at $7.92 \mathrm{GHz}$ with the thickness of $3 \mathrm{~mm}$, outperforming the other ICNF/paraffin composites in Fig. 5a, c. Moreover, the $15 \%$ filler loading composite possesses a broad effective absorption bandwidth of $4.96 \mathrm{GHz}(11.04-16 \mathrm{GHz})$ at $2 \mathrm{~mm}$. The above electromagnetic wave absorption performance is superior to the other outstanding absorbents reported previously, as summarized in Fig. $5 \mathrm{~d}$, suggesting the potential prospect of ICNF as an excellent microwave absorption absorbent with lightweight.

Figure 6 further illustrates the possible electromagnetic wave absorption mechanism of sample ICNF. Firstly, the unique interconnected carbon nanofibers conductive framework endows a lot of conductive pathways, facilitating the migration and the hopping of electrons, then boosting the conductive losses [29,30]. Secondly, the high specific surface area is conductive to multiple reflections and scattering of microwave, which can enhance impedance matching degree, thus inducing a high dielectric loss [31, 32]. Thirdly, nitrogen element in sample ICNF can accelerate the electron transfer and also can improve the dipolar polarization and defect polarization, which are beneficial to the conduction loss and dielectric loss, respectively [33, 34]. Lastly, there are numerous defects and boundaries in sample ICNF, which can give rise to strong dipole orientation relaxation and interfacial polarization relaxation, respectively. 
Fig. 5 Three-dimensional patterns of reflection loss of ICNF/ paraffin composites with filler ratio of $10 \mathrm{wt} \% \mathbf{a}, 15 \mathrm{wt} \% \mathbf{b}$, and 20 wt $\%$ c. d Comparison of microwave performance of sample ICNF with recent reported absorbents


Fig. 6 Schematic illustration of electromagnetic wave absorption mechanism of sample ICNF

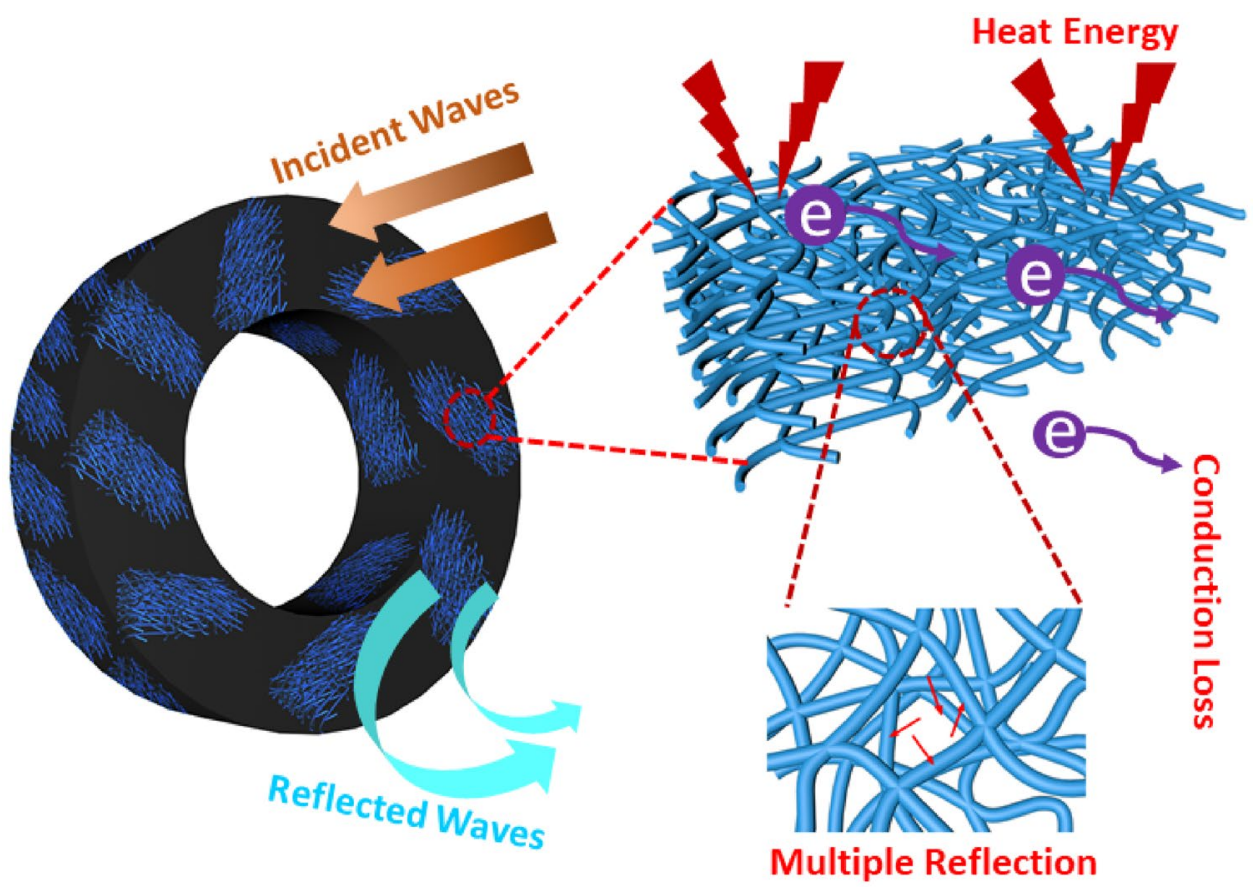

\section{Conclusions}

In summary, three-dimensional interconnected carbon nanofibers derived from fish bone have been successfully fabricated through a facile carbonizing and the following acidizing process. The as-prepared ICNF absorbent exhibits outstanding electromagnetic wave absorption performance with the corresponding minimum reflection loss of $-59.2 \mathrm{~dB}$ at a low loading content of $15 \mathrm{wt} \%$ and the effective bandwidth of $4.96 \mathrm{GHz}$ at a relatively low thickness of $2 \mathrm{~mm}$, outperforming those of most carbonaceous absorbsents reported previously. Moreover, the possible microwave absorption mechanism is discussed, and the excellent microwave properties are mainly attributed to its unique $3 \mathrm{D}$ 
interconnected nanofibers network structure and the doping of nitrogen atoms. These results demonstrate that fish bone is of great potential in the synthesis of efficient and lightweight microwave absorbents by a facile and low-cost strategy.

Acknowledgements The research was financially supported by the Key Natural Science Research Project of Anhui Provincial Education Department (No. KJ2020A0012).

\section{Compliance with ethical standards}

Conflict of interest The author(s) declare that they have no competing interests.

Open Access This article is licensed under a Creative Commons Attribution 4.0 International License, which permits use, sharing, adaptation, distribution and reproduction in any medium or format, as long as you give appropriate credit to the original author(s) and the source, provide a link to the Creative Commons licence, and indicate if changes were made. The images or other third party material in this article are included in the article's Creative Commons licence, unless indicated otherwise in a credit line to the material. If material is not included in the article's Creative Commons licence and your intended use is not permitted by statutory regulation or exceeds the permitted use, you will need to obtain permission directly from the copyright holder. To view a copy of this licence, visit http://creativecommons .org/licenses/by/4.0/.

\section{References}

1. Zeng X, Cheng X, Yu R, Stucky GD (2020) Electromagnetic microwave absorption theory and recent achievements in microwave absorbers. Carbon NY 168:606-623

2. Huang LN, Chen CG, Li ZJ (2020) Challengesandfutureperspectivesonmicrowaveabsorption based on two-dimensional materialsandstructures. Nanotechnology 31:162001

3. Lanre L, Soleimani H, Yahya N, Abbas Z (2020) Recent advances in the development $\mathrm{OF} \mathrm{Fe}_{3} \mathrm{O}_{4}$-BASED microwave absorbing materials. Ceram Int 46:1249-1268

4. Wang X, Liu Y, Han H, Mølhave K, Sun H (2017) Enhanced highfrequency microwave absorption of $\mathrm{Fe}_{3} \mathrm{O}_{4}$ architectures based on porous nanoflake. Ceram Int 43:16013-16017

5. Zhao H et al (2019) Biomass-derived porous carbon-based nanostructures for microwave absorption. Nano Micro Lett 11:1-17

6. Wu Z et al (2018) Hierarchically porous carbons with controlled structures for efficient microwave absorption. J Mater Chem C 6:8839-8845

7. Wang G, Ong SJH, Zhao Y, Xu ZJ, Ji G (2020) Integrated multifunctional macrostructures for electromagnetic wave absorption and shielding. J. Mater. Chem. A 8:24368-24387

8. Zhou M. et al. (2020) Sustainable wood-based composites for microwave absorption and electromagnetic interference shielding. J Mater Chem A 24267-24283.

9. Quan B et al (2020) From intrinsic dielectric loss to geometry patterns: dual-principles strategy for ultrabroad band microwave absorption Nano Res 12(1):7

10. Zhang K, Ye M, Han A, Yang J (2019) Preparation, characterization and microwave absorbing properties of MoS2 and MoS2 -reduced graphene oxide (RGO) composites. J Solid State Chem 277:68-76

11. Ai F et al (2017) Heteroatoms-doped porous carbon derived from tuna bone for high performance Li-S batteries. Electrochim Acta 258:80-89

SN Applied Sciences

APRINGER NATURE journa
12. Guan J, Zhang Z, Ji J, Dou M, Wang F (2017) Hydrothermal synthesis of highly dispersed $\mathrm{Co} 3 \mathrm{O} 4$ nanoparticles on biomass-derived nitrogen-doped hierarchically porous carbon networks as an efficient bifunctional electrocatalyst for oxygen reduction and evolution reactions. ACS Appl Mater Interfaces 9:30662-30669

13. Zhao H, Yeow Seow JZ, Cheng Y, Xu ZJ, Ji G (2020) Green synthesis of hierarchically porous carbons with tunable dielectric response for microwave absorption. Ceram Int 46:15447-15455

14. Li Q et al (2020) Fabrication of porous graphene-like carbon nanosheets with rich doped-nitrogen for high-performance electromagnetic microwave absorption. Appl Surf Sci 530:147298

15. Wacharasindhu S et al (1998) Serum IGF-I and IGFBP-3 levels for normal thai children and their usefulness in clinical practice. J Med Assoc Thail 81:420-430

16. Liu P et al (2021) Magnetic porous N-doped carbon composites with adjusted composition and porous microstructure for lightweight microwave absorbers. Carbon NY 173:655-666

17. Liu P et al (2020) Metal-organic polymer coordination materials derived $\mathrm{Co} / \mathrm{N}$-doped porous carbon composites for frequencyselective microwave absorption. Compos Part B Eng 202:108406

18. Liu P et al (2020) Vacancies-engineered and heteroatoms-regulated $\mathrm{N}$-doped porous carbon aerogel for ultrahigh microwave absorption. Carbon NY 169:276-287

19. Liu P et al (2020) Core-shell Ni@C encapsulated by N-doped carbon derived from nickel-organic polymer coordination composites with enhanced microwave absorption. Carbon N Y 170:503-516

20. Cui $X$ et al (2020) Stable microwave absorber derived from 1D customized heterogeneous structures of $\mathrm{Fe}_{3} \mathrm{~N} @ \mathrm{C}$. Chem Eng J 381:122589

21. Lan D et al (2020) Novel binary cobalt nickel oxide hollowed-out spheres for electromagnetic absorption applications. Chem Eng J 382:122797

22. Liu W, Tan S, Yang Z, Ji G (2018) Hollow graphite spheres embedded in porous amorphous carbon matrix as lightweight and lowfrequency microwave absorbing material through modulating dielectric loss. Carbon NY 138:143-153

23. Liu D et al (2020) MOFs-derived multi-chamber carbon microspheres with enhanced microwave absorption. Carbon N Y 157:478-485

24. Zhao H et al (2018) Reduced graphene oxide decorated with carbon nanopolyhedrons as an efficient and lightweight microwave absorber. J Colloid Interface Sci 528:174-183

25. Duan Y et al (2018) Enhanced electromagnetic microwave absorption property of peapod-like MnO@carbon nanowires. ACS Appl Mater Interfaces 10:40078-40087

26. Qiu X, Wang L, Zhu H, Guan Y, Zhang Q (2017) Lightweight and efficient microwave absorbing materials based on walnut shellderived nano-porous carbon. Nanoscale 9:7408-7418

27. Zhou M et al (2011) Morphology-controlled synthesis and novel microwave absorption properties of hollow urchinlike a-MnO2 nanostructures. J Phys Chem C 115:1398-1402

28. Fu J et al (2017) Enhanced electromagnetic microwave absorption performance of lightweight bowl-like carbon nanoparticles. Ind Eng Chem Res 56:11460-11466

29. Cao WQ, Wang XX, Yuan J, Wang WZ, Cao MS (2015) Temperature dependent microwave absorption of ultrathin graphene composites. J Mater Chem C 3:10017-10022

30. Yang W et al (2020) Production of hierarchical porous carbon nanosheets from cheap petroleum asphalt toward lightweight and high-performance electromagnetic wave absorbents. Carbon NY 166:218-226

31. Wu Z et al (2018) hierarchically porous carbons derived from biomasses with excellent microwave absorption performance. ACS Appl Mater Interfaces 10:11108-11115 
32. Wen B et al (2014) Reduced graphene oxides: light-weight and high-efficiency electromagnetic interference shielding at elevated temperatures. Adv Mater 26:3484-3489

33. Cheng Y, Seow JZY, Zhao H, Xu ZJ, Ji GA (2020) Flexible and lightweight biomass-reinforced microwave absorber. Nano Micro Lett 12:1-15

34. Liang X et al (2020) Environment-stable CoxNiy encapsulation in stacked porous carbon nanosheets for enhanced microwave absorption. Nano-Micro Lett 12:1-12
Publisher's Note Springer Nature remains neutral with regard to jurisdictional claims in published maps and institutional affiliations. 